\title{
Emodin mitigates the oxidative stress induced by cisplatin in osteosarcoma MG63 cells
}

\author{
LI YAN, RUI HU, SONG TU, WEN-JUN CHENG, QIONG ZHENG, \\ JUN-WEN WANG, WU-SHENG KAN and YI-JUN REN
}

\begin{abstract}
Department of Reparative and Reconstructive Surgery of Orthopedics, Puai Hospital, Tongji Medical College, Huazhong University of Science and Technology, Wuhan, Hubei 430033, P.R. China
\end{abstract}

Received December 4, 2014; Accepted October 12, 2015

DOI: $10.3892 / 01.2016 .4902$

\begin{abstract}
Previously, the application of cisplatin in chemotherapy was limited due to the significant side effects on normal cell growth. In the present study, the concomitant application of emodin with cisplatin was demonstrated to ameliorate cisplatin-induced oxidative stress and markedly suppress tumor cell proliferation for the first time. Human osteosarcoma MG-63 cells were treated with cisplatin alone or in combination with emodin. The cell viability was determined by MTS assays and the augmentation of reactive oxygen species were determined by fluorogenic probes; in addition, a stable MG-63 subline bearing antioxidant response element (ARE)-driven luciferase expression was developed to monitor the activation of the nuclear factor erythroid 2-related factor 2 (Nrf2)-ARE signaling pathway. The results indicated that cisplatin or emodin may inhibit MG-63 cell proliferation in a time- or dose-dependent manner, respectively. Concomitant treatment with cisplatin and emodin demonstrated synergic anti-tumor effects. Cisplatin augmented reactive oxygen species in the MG-63 cells, followed by the translocation of Nrf2 from the cytoplasm into the nucleus, which triggered ARE-driven luciferase expression. The addition of emodin diminished the previously described phenomenon, resulting in decreased ROS augmentation, translocation of Nrf2 and ARE-driven luciferase activity. In conclusion, emodin could ameliorate cisplatin-induced oxidative stress and protect the cells from oxidative stress-induced damage. The findings of the present study provide a novel strategy for the treatment of osteosarcoma using emodin and cisplatin.
\end{abstract}

Correspondence to: Dr Yi-Jun Ren, Department of Reparative and Reconstructive Surgery of Orthopedics, Puai Hospital, Tongji Medical College, Huazhong University of Science and Technology, 473 Han Zheng Street, Wuhan, Hubei 430033, P.R. China

E-mail: renyijjpuai@yeah.net

Key words: emodin, cisplatin, oxidative stress, osteosarcomas

\section{Introduction}

Cisplatin is a chemotherapy drug that is widely used to treat various cancers, including bladder (1), ovarian (2), breast (3), and pancreatic cancers (4), and osteosarcoma (5). Cisplatin is a highly reactive compound that interferes with cell division by mitosis and cross-linking DNA (6). When the DNA repair mechanism fails to restore the integrity of genomic DNA, the apoptosis pathway is activated in turn (7). The presence of DNA damage-mediated cell death elucidates the high toxicity of cisplatin for fast-dividing cells. Nevertheless, cisplatin also demonstrates multiple side effects on normal tissues and non-dividing cells, including kidney, ear and sensory nerves (8-10), which suggests that the mechanism of cisplatin-induced DNA damage may not explain the entire story and that the underlying pathways remain poorly defined. Previous research demonstrates that cisplatin accumulates in the mitochondria, damaging mitochondrial DNA and proteins (11), and leads to the increase of an oxidative stress reaction in normal cells $(12,13)$, suggesting that oxidative stress is involved in the pathogenesis of cisplatin-induced cytotoxicity. Nuclear factor erythroid 2-related factor 2 (Nrf2) is pivotal for modulating cellular redox homeostasis and aberrant Nrf2 expression is commonly observed in numerous cancer cells; furthermore, Nrf2/antioxidant response element (ARE) signaling is closely associated with tumor cell resistance to chemotherapeutic drugs (14).

Numerous endogenous antioxidants, including superoxide and dismutase catalase, are reported to block oxidative stress $(15,16)$, with each possessing the capability of scavenging reaction oxygen species (ROS). Deficiency of these enzymes may worsen the oxidative stress-induced cell damage. Worldwide, studies are devoted to identifying exogenous antioxidants that ameliorate oxidative stress conditions. For example, curcumin, isolated from the rhizome of Curcuma longa, demonstrates antioxidant abilities in vivo (17). Emodin, another natural anthraquinone, demonstrates protective or ameliorative effects against inflammation or oxidative stress-associated diseases $(18,19)$. The aim of the present study is to assess the ability of emodin to alleviate the oxidative stress induced by cisplatin, and the results may elucidate the benefits of combined chemotherapy using emodin and cisplatin. 


\section{Materials and methods}

Drugs and reagents. Cisplatin was supplied by Qilu Pharmaceutical Co., Ltd. (Jinan, Shandong, China) and was prepared in phosphate-buffered saline (PBS) prior to use. Emodin (catalog no., A7687) was obtained from Sigma-Aldrich (St. Louis, MO, USA) and was dissolved in dimethyl sulfoxide. Unless specified, all reagents for cell culture were either Gibco or Invitrogen products obtained from (Thermo Fisher Scientific, Inc., Waltham, MA USA). Mouse anti-human monoclonal Nrf2 (catalog no., sc-365949) and $\beta$-actin (catalog no., sc-1616) primary antibodies were from Santa Cruz Biotechnology (Dallas, TX, USA). ONEGlo reagent (catalog no., E6110) for the detection of firefly luciferase activity was purchased from Promega Corporation (Madison, WI, USA).

Cell line and culture conditions. Human osteosarcoma MG-63 cells were obtained from American Type Culture Collection (Manassas, VA, USA) and cultured at $37^{\circ} \mathrm{C}$ in a humidified atmosphere containing 5\% $\mathrm{CO}_{2}$ in Eagle's minimal essential medium complete medium supplemented with $10 \%$ fetal bovine serum.

Cell proliferation assay using MTS. The cell proliferation assay was conducted using a colorimetric method with an MTS assay kit (CellTiter $96^{\circledR}$ Aqueous Non-Radioactive Cell Proliferation Assay; Promega Corporation, Madison, WI, USA). In brief, the MG-63 cells in the logarithmic phase of growth were seeded into a 96-well plate at a density of $1 \times 10^{4}$ cells per well, $24 \mathrm{~h}$ prior to treatment. The cells were then treated with cisplatin $(0.1,0.2,0.4,0.8,1.6,3.2 \mu \mathrm{M})$ alone or in combination with emodin $(5,10,20,40,80$ and $100 \mu \mathrm{M})$ at the indicated concentration for 24,36 and $48 \mathrm{~h}$, respectively. The production of formazan was read at $490 \mathrm{~nm}$ using Flexstation 3 (Molecular Devices, Sunnyvale, CA, USA). The intensity of the color produced was proportional to the number of living cells.

Detection of intracellular reactive oxygen species. The ROS in live cells were determined using a fluorogenic probe (CellROX ${ }^{\circledast}$ Oxidative Stress Reagents; catalog no., C10422; Molecular Probes Life Technologies, Carlsbad, CA, USA). The assay procedure was performed as follows. Briefly, the MG-63 cells were plated in a $6-\mathrm{cm}$ plate and treated with cisplatin $(0.1,0.2,0.4,0.8,1.6,3.2 \mu \mathrm{M})$ alone or in combination with emodin $(5,10,20,40,80$ and $100 \mu \mathrm{M})$ at the indicated concentration. The cells were then stained with $5 \mu \mathrm{M}$ CellROX $^{\circledR}$ Deep Red Reagent, by adding the complete probe to the complete medium and incubating the cells at $37^{\circ} \mathrm{C}$ for $30 \mathrm{~min}$. The cells were then washed using PBS and analyzed using BD FACSCalibur (BD Biosciences, Franklin Lakes, NJ, USA).

Western blot analysis. For immunoblot analysis, the cells treated with cisplatin or emodin plus cisplatin were washed with PBS and then lysed using radioimmunoprecipitation assay buffer. Subsequent to centrifugation at $12,000 \mathrm{x}$ g for $15 \mathrm{~min}$ at $4^{\circ} \mathrm{C}$, the supernatant was separated on a $10 \%$ sodium dodecyl sulfate polyacrylamide gel electrophoresis gel and transblotted onto a polyvinylidene fluoride membrane. Subsequent to blocking with $5 \%$ bovine serum albumin in Tris-buffered saline with Tween-20, the membrane was incubated with aforementioned Nrf2 and $\beta$-actin (catalog no., sc-1616; Santa Cruz Biotechnology) antibodies at dilutions of 1:3,000, and then with the fluorescein-labeled rabbit anti-mouse polyclonal secondary antibody (catalog no., ab6728; Santa Cruz Biotechnology) at a dilution of 1:2,000. Specific bands were visualized using Li-COR Odyssey (LI-COR Biosciences, Lincoln, NE, USA).

Generation of the stable reporter cell line and ARE-luciferase activity assay. The ARE-luciferase reporter plasmid was constructed using a pGL3 basic vector containing the full-length firefly luciferase gene. The sequence of the minimal thymidine kinase (TK) promoter, which was coupled to eight ARE (8xARE) repeats, was inserted upstream of the open reading frame of luciferase. The plasmid was sequenced to verify the accuracy of the inserts, with the sequence of the minimal TK promoter coupled to 8xARE repeats as follows: GTGACAAAGCACCCGTGACAAAGCACCCGTGACAAA GCACCCGTGACAAAGCACCCGGACAAAGCACCCGTG ACAAAGCACCCGTGACAAAGCACCCGTGACAAGCAT TCGCATATTAAGGTGACGCGTGTGGCCTCGAACACCG AGCGACCCTGCAGCGACCCGCTTAA.

The pGL-6xARE plasmid containing the neomycin selectable marker was stably transfected into the MG- 63 cells using electrotransformation (Cell Line Nucleofector ${ }^{\circledR}$ kit C; catalog no., VCA-1004; Lonza Group, Ltd., Basel, Switzerland). The stable clones were screened using G418-containing media for 3-4 weeks.

To investigate the luciferase activity, the stable MG-63 cells were seeded into a 96-well plate at a density of $2 \times 10^{4}$ cells per well in the conditional growth medium $(50 \mu \mathrm{l}$ Dulbecco's modified Eagle medium; catalog no., 11965118; Gibco, Thermo Fisher Scientific, Inc.) and incubated overnight at $37^{\circ} \mathrm{C}$. The culture medium was then replaced with fresh medium containing cisplatin $(0.1,0.2,0.4,0.8,1.6,3.2 \mu \mathrm{M})$ alone or cisplatin plus emodin $(5,10,20,40,80$ and $100 \mu \mathrm{M})$ at the indicated concentration for $24 \mathrm{~h}$. Firefly luciferase activity was determined by ONEGlo reagent (Promega Corporation, Madison, WI, USA).

Statistical analysis. The statistical analysis was performed using GraphPad Prism (version 6.0; GraphPad Software Inc., San Diego, CA, USA). The results were expressed as the mean \pm standard error of the mean. Comparisons between groups were performed using one-way analysis of variance. Student's unpaired $t$-test was used and $\mathrm{P}<0.05$ was considered to indicate a statistically significant difference.

\section{Results}

Combination of cisplatin with emodin enhanced the inhibitory effects on MG-63 cell proliferation. The effects of cisplatin or emodin on human osteosarcoma MG-63 cell growth were studied. First, the $\mathrm{IC}_{50}$ of a single treatment with cisplatin or emodin was determined by incubating the cells with a series of drug concentrations and then measuring the cell survival rate at 6 days post-treatment (Fig. 1A and B). Then, the time course of single cisplatin or emodin, as well as the combination of 

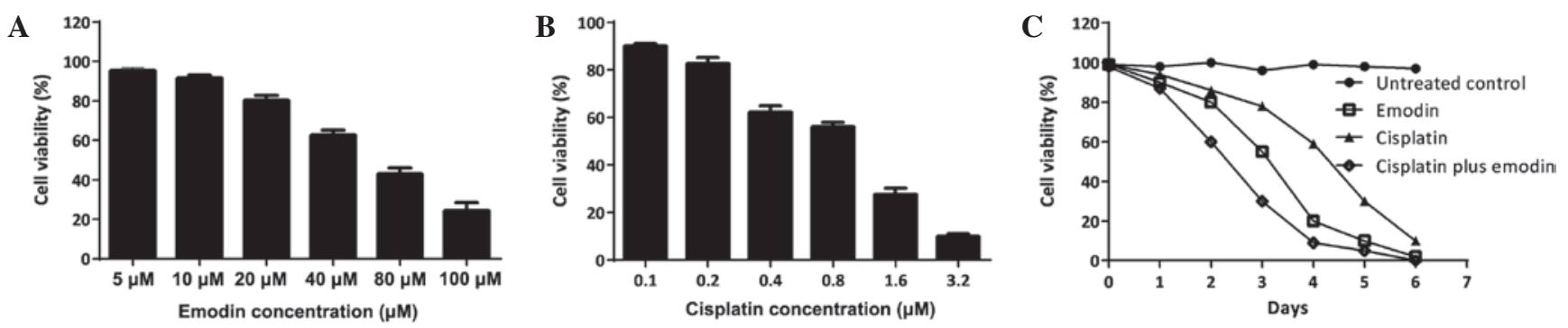

Figure 1. Inhibitory effects of cisplatin or emodin on MG-63 cells. MG-63 cells were incubated with cisplatin or emodin at the indicated concentrations. Fig. 1 demonstrates that the growth of MG-63 cells was suppressed by (A) emodin and (B) cisplatin in dose-dependent manner. (C) MG-63 proliferation was inhibited time-dependently by cisplatin (black triangle), emodin (empty square), or combination treatment (diamonds). Cell survival was expressed relative to untreated control cells and the data were expressed as the mean values with the standard deviation from three independent experiments each performed in triplicate.
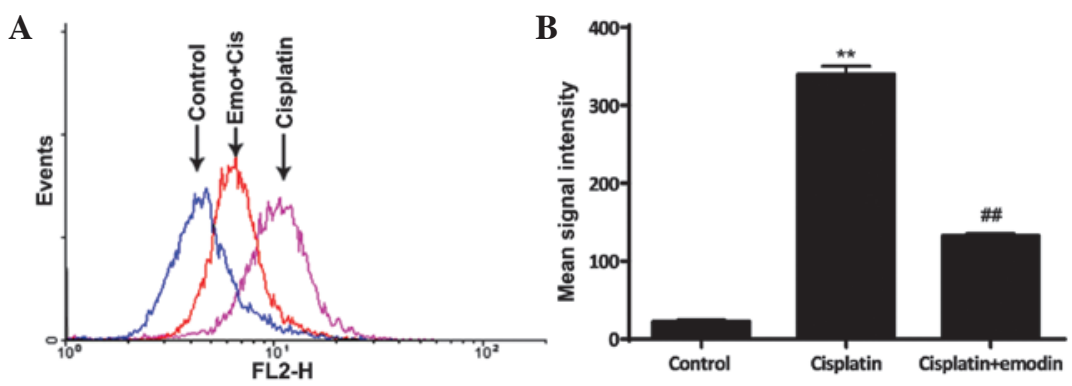

Figure 2. Effects of cisplatin and emodin for accumulating or reducing ROS in MG-63 cells. (A) The accumulation of ROS in live cells was detected using the CellROX $^{\circledR}$ Red reagent fluorogenic probe, which released bright red photostable fluorescence upon oxidation by reactive oxygen species, and the accumulation was measured by flow cytometry. Cells were co-stained with Annexin V and analyzed by flow cytometry. (B) Statistical analysis for the fluorescence. ${ }^{* * *} \mathrm{P}<0.01$ represents the signal intensity in the cisplatin group compared to the control group; ${ }^{\# \#} \mathrm{P}<0.01$ represents the signal intensity in the cisplatin + emodin group compared to the cisplatin group. ROS, reactive oxygen species.
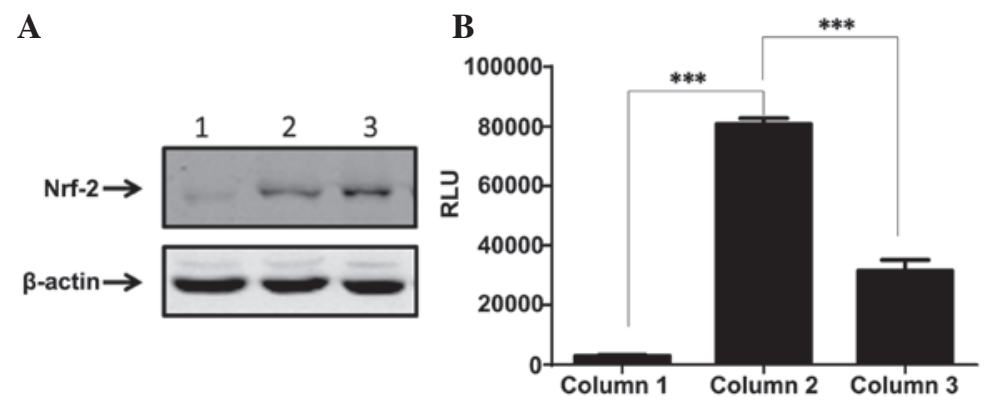

Figure 3. Effect of cisplatin or emodin on the translocation of Nrf2 from the cytoplasm to the nucleus. (A) MG-63 cells were treated with single reagent of cisplatin or in combination with emodin and the nuclear fraction was isolated and analyzed for Nrf2 expression using western blot analysis. Lane 1, Untreated MG-63 parental cells; Lane 2, MG-63 cells treated with cisplatin; Lane 3, MG-63 cells treated with cisplatin and emodin. (B) Nrf2 expression was also analyzed and compared. Column 1, MG-63/ARE-Luciferase reporter cell line; Column 2, Cisplatin-induced MG-63/ARE-luciferase reporter cells; Column 3, Cisplatin-emodin treated MG-63/ARE-luciferase reporter cells. Nrf2, nuclear factor erythroid 2-related factor 2; ARE, antioxidant response element; RLU, relative light unit. ${ }^{* * * *} \mathrm{P}<0.001$ for RLU of column 2 vs. column 1 and for column 2 vs column 3.

these two drugs, was assessed using a single dose (Fig. 1C). The data demonstrated that cisplatin and emodin could efficiently suppress the proliferation of MG-63 cells in a dose-dependent manner. The administration of a single dose of $0.2 \mu \mathrm{M}$ cisplatin or $10 \mu \mathrm{M}$ emodin inhibited MG-63 cell growth time-dependently; and using a combination of cisplatin with emodin strengthened the inhibitory effects on MG-63 growth, demonstrating synergistic function.

Emodin attenuated cisplatin-induced oxidative stress in MG-63 cells. ROS was important in the pathogenesis of cisplatin-induced cell damage. The data demonstrated that the intracellular ROS level was significantly increased subsequent to the treatment of the cells with $0.1 \mu \mathrm{M}$ cisplatin for $24 \mathrm{~h}(\mathrm{P}<0.01$; Fig. 2). However, ROS accumulation was suppressed when the cells were simultaneously treated with $0.1 \mu \mathrm{M}$ cisplatin and $5 \mu \mathrm{M}$ emodin, when compared with the single treatment of cisplatin $(\mathrm{P}<0.01$; Fig. 2$)$. These results suggested that emodin prevents cisplatin-induced ROS accumulation in MG-63 cells.

Emodin and cisplatin changed oxidative stress conditions through the Nrf2/ARE signaling pathway. Nrf2 regulated cytoprotective genes that contained ARE in the promoter region. 
An ARE-driven firefly luciferase reporter MG-63 stable subline was developed, as previously reported (20). Nrf2 may be retained in cytoplasm under normal conditions, but may be translocated into the nucleus when the cells are switched to oxidative stress conditions. The nuclear faction of the cells was isolated and analyzed using western blot analysis (Fig. 3A). The data demonstrated that $\mathrm{Nrf} 2$ expression in the nucleus was dramatically improved by treatment with cisplatin, while emodin mildly blocked the cisplatin-induced translocation of Nrf2 (P<0.001; Fig. 3B). To investigate whether the Nrf2/ARE pathway was fully activated, the ARE/luciferase reporter MG-63 cells were tested with single cisplatin treatment or in combination with emodin. The results demonstrated that cisplatin triggered the cascade of Nrf2-ARE, resulting in the robust elevation of luciferase activity. Emodin counteracted the function of cisplatin to a certain extent. These data indicated that emodin mitigated cisplatin-induced cell damage by blocking the translocation of Nrf2 into the nucleus.

\section{Discussion}

The present study investigated the function of emodin in attenuating cisplatin-induced oxidative stress in human osteosarcoma MG-63 cells. The findings demonstrated that the combined application of emodin and cisplatin inhibited the malignant properties of MG-63 cells, further suppressing cellular proliferation when compared with a single treatment with cisplatin. In addition, the oxidative stress pathway, which was activated by cisplatin treatment, was reversed by the addition of emodin, demonstrating the clinical relevance of using emodin and cisplatin simultaneously for novel therapeutic strategies for the treatment of osteosarcoma. Emodin plus cisplatin inhibits tumor cell growth and ameliorates cisplatin-induced oxidative stress.

As a natural bioactive anthraquinone, emodin has previously been used therapeutically to treat gastroenteric and liver diseases (21). Emodin is widely used for multiple effects, such as antiviral and hepatoprotective activities $(22,23)$; however, current studies focus more on the anti-tumor activity of emodin. Previously, emodin was demonstrated to inhibit breast cancer cell growth (24). Therefore, it was possible that emodin may arrest human osteosarcoma proliferation. The present study identified that emodin could suppress MG-63 cell growth in a time- and dose-dependent manner. Furthermore, concomitant incubation of MG-63 cells with cisplatin and emodin demonstrated higher cellular susceptibility when compared with single treatment with cisplatin or emodin, indicating synergistic effects.

ROS have been demonstrated to suppress the proliferation of malignant cells and even induce cellular apoptosis (6). Cisplatin may induce the ROS response and alter the redox status in malignant and normal cells, which may limit the clinical applications of cisplatin (25). The present study also demonstrated the augmentation of ROS in cisplatin-treated MG-63 cells, as previously reported in another type of cell model (19). Waly et al demonstrated that emodin protected normal cells from cisplatin-induced oxidative stress (26). The findings of the present study were in accordance with the outcomes of the study by Waly et al. Furthermore, in the present study, the concomitant application of emodin with cisplatin exerted synergistic effects on osteosarcoma cell proliferation, although emodin counteracted the oxidative stress conditions induced by cisplatin.

In addition to the experiments involving the blocking of cell proliferation, the present study also investigated how emodin manipulated the oxidative stress signaling pathway. Previously, Nrf2 regulated the expression of multiple oxidative stress-associated genes (27), and upon stimulation with certain inducers, Nrf2 was translocated into the nucleus, where Nrf2 activated the ARE-driven antioxidant and detoxification genes. This classical oxidative stress signaling pathway was hypothesized to be modulated by certain anticancer drugs. In the present study, cisplatin treatment was found to carry Nrf2 from the cytoplasm into the nucleus and trigger the ARE-driven luciferase expression, while concomitant treatment with emodin diminished the translocation of Nrf2.

The molecular mechanism behind the present findings remains undefined in detail, but the present study indicates that the combined application of emodin with cisplatin may be a novel therapeutic strategy for the treatment of osteosarcoma.

\section{References}

1. Shirato A, Kikugawa T, Miura N, Tanji N, Takemori N, Higashiyama $\mathrm{S}$ and Yokoyama $\mathrm{M}$ : Cisplatin resistance by induction of aldo-keto reductase family 1 member $\mathrm{C} 2$ in human bladder cancer cells. Oncol Lett 7: 674-678, 2014.

2. Muggia F: Platinum compounds 30 years after the introduction of cisplatin: Implications for the treatment of ovarian cancer. Gynecol Oncol 112: 275-281, 2009.

3. Heinemann V: Gemcitabine plus cisplatin for the treatment of metastatic breast cancer. Clin Breast Cancer 3 (Suppl 1): 24-29, 2002.

4. Elligers KT, Davies M, Sanchis D, Ferencz T and Saif MW: Rechallenge with cisplatin in a patient with pancreatic cancer who developed a hypersensitivity reaction to oxaliplatin. Is skin test useful in this setting? JOP 9: 197-202, 2008.

5. Zhao J, Wang M, Li Z, Chen J, Yin Z, Chang J, Gao D and Wang S: Interferon- $\alpha$ suppresses invasion and enhances cisplatin-mediated apoptosis and autophagy in human osteosarcoma cells. Oncol Lett 7: 827-833, 2014.

6. Davis W Jr, Ronai Z and Tew KD: Cellular thiols and reactive oxygen species in drug-induced apoptosis. J Pharmacol Exp Ther 296: 1-6, 2001.

7. Casares C, Ramirez-Camacho R, Trinidad A, Roldán A, Jorge E and Garcia-Berrocal JR: Reactive oxygen species in apoptosis induced by cisplatin: Review of physiopathological mechanisms in animal models. Eur Arch Otorhinolaryngol 269: 2455-2459, 2012.

8. Kursunluoglu G, Kayali HA and Taskiran D: The effect of cisplatin toxicity and capsaicin on electron transport chain in liver and kidney of sprague dawley rats. Cell Biochem Biophys 69: 707-16, 2014.

9. Leinung M, Cuny C, Diensthuber M, Stöver T and Wagenblast J: Small molecules in combination with conventional chemotherapeutic drugs: Light at the end of the tunnel? Oncol Lett 4: 1043-1046, 2012.

10. Slattery EL and Warchol ME: Cisplatin ototoxicity blocks sensory regeneration in the avian inner ear. J Neurosci 30: 3473-3481, 2010.

11. Rodrigues MA, Rodrigues JL, Martins NM, Barbosa F, Curti C, Santos NA and Santos AC: Carvedilol protects against cisplatin-induced oxidative stress, redox state unbalance and apoptosis in rat kidney mitochondria. Chem Biol Interact 189: 45-51, 2011.

12. Dehne N, Lautermann J, Petrat F, Rauen U and de Groot H: Cisplatin ototoxicity: Involvement of iron and enhanced formation of superoxide anion radicals. Toxicol Appl Pharmacol 174: 27-34, 2001.

13. Jiang Y, Guo C, Vasko MR and Kelley MR: Implications of apurinic/apyrimidinic endonuclease in reactive oxygen signaling response after cisplatin treatment of dorsal root ganglion neurons. Cancer Res 68: 6425-6434, 2008. 
14. Lau A, Villeneuve NF, Sun Z, Wong PK and Zhang DD: Dual roles of Nrf2 in cancer. Pharmacol Res 58: 262-270, 2008.

15. Kessova IG,Ho YS, Thung S and Cederbaum AI: Alcohol-induced liver injury in mice lacking $\mathrm{Cu}, \mathrm{Zn}$-superoxide dismutase. Hepatology 38: 1136-1145, 2003 .

16. Kim SJ, Lee JW, Jung YS, Kwon do Y, Park HK, Ryu CS, Kim SK, Oh GT and Kim YC: Ethanol-induced liver injury and changes in sulfur amino acid metabolomics in glutathione peroxidase and catalase double knockout mice. J Hepatol 50: 1184-1191, 2009.

17. M Khopde S, Priyadarsini KI, Venkatesan P and Rao MN: Free radical scavenging ability and antioxidant efficiency of curcumin and its substituted analogue. Biophys Chem 80: 85-91, 1999.

18. Lin KY and Uen YH: Aloe-emodin, an anthraquinone, in vitro inhibits proliferation and induces apoptosis in human colon carcinoma cells. Oncol Lett 1: 541-547, 2010.

19. Yon JM, Baek IJ, Lee BJ, Yun YW and Nam SY: Emodin and [6]-gingerol lessen hypoxia-induced embryotoxicities in cultured mouse whole embryos via upregulation of hypoxia-inducible factor $1 \alpha$ and intracellular superoxide dismutases. Reprod Toxicol 31: 513-518, 2011

20. Wang XJ, Hayes JD and Wolf CR: Generation of a stable antioxidant response element-driven reporter gene cell line and its use to show redox-dependent activation of nrf2 by cancer chemotherapeutic agents. Cancer Res 66: 10983-10994, 2006.

21. Peigen X, Liyi H and Liwei W: Ethnopharmacologic study of Chinese rhubarb. J Ethnopharmacol 10: 275-293, 1984.
22. Andersen DO, Weber ND, Wood SG, Hughes BG, Murray BK and North JA: In vitro virucidal activity of selected anthraquinones and anthraquinone derivatives. Antiviral Res 16: 185-196, 1991

23. Arosio B, Gagliano N, Fusaro LM, Parmeggiani L, Tagliabue J, Galetti P, De Castri D, Moscheni C and Annoni G: Aloe-Emodin quinone pretreatment reduces acute liver injury induced by carbon tetrachloride. Pharmacol Toxicol 87 : 229-233, 2000.

24. Huang PH, Huang CY, Chen MC, Lee YT, Yue CH, Wang HY and Lin H: Emodin and aloe-emodin suppress breast cancer cell proliferation through ER $\alpha$ inhibition. Evid Based Complement Alternat Med 2013: 376123, 2013.

25. Stewart JH IV, Tran TL, Levi N, Tsai WS, Schrump DS and Nguyen DM: The essential role of the mitochondria and reactive oxygen species in cisplatin-mediated enhancement of rats fas ligand-induced apoptosis in malignant pleural mesothelioma. J Surg Res 141: 120-131, 2007.

26. Waly MI, Ali BH, Al-Lawati I and Nemmar A: Protective effects of emodin against cisplatin-induced oxidative stress in cultured human kidney (HEK 293) cells. J Appl Toxicol 33: 626-630, 2013.

27. Ge M, Chi X, Zhang A, Luo G, Sun G, Xie H and Hei Z: Intestinal NF-E2-related factor-2 expression and antioxidant activity changes in rats undergoing orthotopic liver autotransplantation. Oncol Lett 6: 1307-1312, 2013. 